\title{
Arbitrary pole placement with the extended Kautsky-Nichols-van Dooren parametric form with minimum gain
}

\author{
Robert Schmid, Thang Nguyen and Lorenzo Ntogramatzidis
}

\begin{abstract}
We consider the classic problem of pole placement by state feedback. We revisit the well-known eigenstructure assignment algorithm of Kautsky, Nichols and van Dooren [1] and extend it to obtain a novel parametric form for the pole-placing feedback matrix that can deliver any set of desired closed-loop eigenvalues, with any desired multiplicities. This parametric formula is then employed to introduce an unconstrained nonlinear optimisation algorithm to obtain a feedback matrix that delivers the desired pole placement with minimum gain.
\end{abstract}

\section{INTRODUCTION}

We consider the classic problem of repeated pole placement for linear time-invariant (LTI) systems in state space form

$$
\dot{x}(t)=A x(t)+B u(t),
$$

where, for all $t \in \mathbb{R}, x(t) \in \mathbb{R}^{n}$ is the state and $u(t) \in \mathbb{R}^{m}$ is the control input, and $A$ and $B$ are appropriate dimensional constant matrices. We assume that $B$ has full column-rank, and that the pair $(A, B)$ is reachable. We let $\mathscr{L}=\left\{\lambda_{1}, \ldots, \lambda_{v}\right\}$ be a self-conjugate set of $v \leq n$ complex numbers, with associated algebraic multiplicities $\mathscr{M}=\left\{m_{1}, \ldots, m_{v}\right\}$ satisfying $m_{1}+\cdots+m_{v}=n$. The problem of arbitrary exact pole placement $(E P P)$ by state feedback is that of finding a real gain matrix $F$ such that the closed-loop matrix $A+B F$ has eigenvalues given by the set $\mathscr{L}$ with multiplicities given by $\mathscr{M}$, i.e., $F$ satisfies the equation

$$
(A+B F) X=X \Lambda,
$$

where $\Lambda$ is a $n \times n$ Jordan matrix obtained from the eigenvalues of $\mathscr{L}$, including multiplicities, and $X$ is a matrix of closed-loop eigenvectors of unit length. The matrix $\Lambda$ can be expressed in the Jordan (complex) canonical form

$$
\Lambda=\operatorname{blkdiag}\left\{J\left(\lambda_{1}\right), J\left(\lambda_{2}\right), \cdots, J\left(\lambda_{v}\right)\right\}
$$

where each $J\left(\lambda_{i}\right)$ is a Jordan matrix for $\lambda_{i}$ of order $m_{i}$, and may be composed of $g_{i}$ mini-blocks

$$
J\left(\lambda_{i}\right)=\operatorname{blkdiag}\left\{J_{1}\left(\lambda_{i}\right), J_{2}\left(\lambda_{i}\right), \cdots, J_{g_{i}}\left(\lambda_{i}\right)\right\}
$$

where $g_{i} \leq m$. We use $\mathscr{P} \stackrel{\text { def }}{=}\left\{p_{i, k} \mid 1 \leq i \leq v, 1 \leq k \leq g_{i}\right\}$ to denote the orders of the Jordan mini-blocks $J_{k}\left(\lambda_{i}\right)$ that comprise $J\left(\lambda_{i}\right)$. It is well-known that when $(A, B)$ is a reachable pair,

Robert Schmid is with the Department of Electrical and Electronic Engineering, University of Melbourne, Australia. E-mail: rschmideunimelb.edu. au

Thang Nguyen is with the Department of Engineering, University of Exeter, UK. E-mail: T.Nguyen-Tien@exeter.ac.uk

Lorenzo Ntogramatzidis is with the Department of Mathematics and Statistics, Curtin University, Australia. E-mail: L.Ntogramatzidis@curtin.edu. au arbitrary multiplicities of the closed-loop eigenvalues can be assigned by state feedback, but the possible orders of the associated Jordan structures are constrained by the system controllability indices (or Kronecker invariants) [2]. If $\mathscr{L}$, $\mathscr{M}$ and $\mathscr{P}$ satisfy the conditions of the Rosenbrock theorem, we say that the triple $(\mathscr{L}, \mathscr{M}, \mathscr{P})$ defines an admissible Jordan structure for $(A, B)$.

In order to consider optimal selections for the gain matrix, it is important to have a parametric formula for the set of gain matrices that deliver the desired pole placement, and numerous such parameterisations have appeared in the literature over the past three decades. In [1], a method for obtaining suitable $F$ was introduced involving a QRfactorisation for $B$ and a Sylvester equation for $X$, which requires $\Lambda$ in (2) to be a diagonal matrix. In particular this means that the desired multiplicities must satisfy $m_{i} \leq m$ for all $i \in\{1, \ldots, v\}$. Both the widely-used MATLAB ${ }^{\circledR}$ routine place.m and the MATHEMATICA ${ }^{\circledR}$ routine KNVD are based on the algorithm proposed in [1]. In [3] this method was used to develop a parametric formula for $X$ and $F$, in terms of a suitable parameter matrix; we discuss this method in detail in Section II.

Other parameterisations have been presented in the literature that do not impose a constraint on the multiplicity of the eigenvalues to be assigned. In [4] a procedure was given for obtaining the gain matrix by solving a Sylvester equation in terms of an $n \times m$ parameter matrix, provided the closed-loop eigenvalues do not coincide with the open-loop ones. In [5] a parametric form is presented in terms of the inverses of the matrices $A-\lambda_{i} I_{n}$ (where $I_{n}$ denotes the $n \times n$ identity matrix), which also requires the assumption that the closedloop eigenvalues are all distinct from the open-loop ones.

More recently, in [6] the parametric formula of [1] was revisited for the case where $\Lambda$ was any admissible Jordan matrix, and a parameterisation was obtained for the pole placing matrix $F$ by using the eigenvector matrix $X$ as a parameter. The case where $\mathscr{L}$ contains any desired closedloop eigenvalues and multiplicities is also considered in [7], where a parametric form for $F$ is presented in terms of the solution to a Sylvester equation, also using the eigenvector matrix $X$ as a parameter. However, maximum generality in these parametric formulae has been achieved at the expense of efficiency. Where methods [1], [4], [5] all employ parameter matrices of dimension $m \times n$, the parameter matrices in [6] and [7] have dimension $n \times n$.

In our recent papers [8]-[9], we gave a novel parametric form for $X$ and $F$ based on the famous pole placement algorithm of Moore [10]. This parameterisation employed parameter 
matrices of dimension $m \times n$, but required $\Lambda$ to be diagonal, and hence also assumes the closed-loop eigenvalues have multiplicities of at most $m$. Very recently in our papers [11][12] we generalized this parametric form to accommodate arbitrary multiplicities; the method was based on the pole placement method of Klein and Moore [13]. The principal merit of this approach was to obtain a parameterisation that combines the generality of [6] and [7] with the computational efficiency that comes from an $m \times n$ dimensional parameter matrix.

The first aim of this paper is to revisit the pole-placing feedback method of [1] and generalise it to obtain a parametric formula that can assign arbitrary pole-placement. For a suitable real or complex $m \times n$ parameter matrix $K$, we obtain the eigenvector matrix $X(K)$ and gain matrix $F(K)$ by building the Jordan chains starting from the selection of eigenvectors from the kernel of certain matrix pencils, and thus avoid the need for matrix inversions, or the solution of Sylvester matrix equations. Thus the results of this paper neatly parallel the achievements of [11] in providing a new parametric form to achieve pole placement with arbitrary multiplicities, while employing an $m \times n$-dimensional parameter matrix.

The second aim of the paper is to employ this novel parametric form to seek the solution to the minimum gain exact pole placement problem (MGEPP), which involves solving the EPP problem and also obtaining the feedback matrix $F$ that has the smallest gain, which is of as a measure of the control amplitude or energy required. In [14] the MGEPP problem is addressed for the specific case of placing multiple deadbeat modes with minimum Frobenius gain. Recently the general problem of assigning any desired set of poles with any desired multiplicities with minimum Frobenius gain has been considered in [15].

Finally, we demonstrate the performance of the resulting algorithm by considering an example involving the assignment of deadbeat modes, and compare the performance against the methods of [11], [14], [15]. We see that the methods introduced in this paper are able to deliver the desired eigenstructure with equivalent or smaller gain than these alternative methods.

We begin with some definitions and notation. We say that $\mathscr{L}$ is $\sigma$-conformably ordered if there an integer $\sigma$ such that the first $2 \sigma$ values of $\mathscr{L}$ are complex while the remaining are real, and for all odd $k \leq 2 \sigma$ we have $\lambda_{k+1}=\bar{\lambda}_{k}$. For example, the set $\mathscr{L}=\{10 j,-10 j, 2+2 j, 2-2 j, 7\}$ is 2 conformably ordered. Notice that, since $\mathscr{L}$ is symmetric, we have $m_{i}=m_{i+1}$ for odd $i \leq \sigma$. In the following we implicitly assume that an admissible Jordan structure $(\mathscr{L}, \mathscr{M}, \mathscr{P})$ is $\sigma$ conformably ordered, for some integer $\sigma$. For any matrix $X$ we use $X(l)$ to denote the $l$-th column of $X$. The symbol $0_{n}$ represents the zero vector of length $n$, and $I_{n}$ is the $n$ dimensional identity matrix.

Let $X$ denote any complex matrix partitioned into submatrices $X=\left[X_{1} \ldots X_{V}\right]$ ordered such that any complex submatrices occur consecutively in complex conjugate pairs, and so that, for some integer $s$, the first $2 s$ submatrices are complex while the remaining are real. We define a real matrix
$\mathfrak{R e}\{X\}$ of the same dimension as $X$ thus: if $X_{i}$ and $X_{i+1}$ are consecutive complex conjugate submatrices of $X$, then the corresponding submatrices of $\mathfrak{R e}\{X\}$ are $\frac{1}{2}\left(X_{i}+X_{i+1}\right)$ and $\frac{1}{2 j}\left(X_{i}-X_{i+1}\right)$.

\section{Pole Placement Methods}

We now revisit the algorithm of [1] for the gain matrix $F$ that solves the exact pole placement problem (2), for the case where $\Lambda$ is a diagonal matrix.

Theorem 2.1: ([1], Theorem 3) Given $\Lambda=$ $\operatorname{diag}\left\{\lambda_{1}, \lambda_{2}, \ldots, \lambda_{n}\right\}$ and $X$ non-singular, then there exists $F$, a solution to (2) if and only if

$$
U_{1}^{\top}(A X-X \Lambda)=0
$$

where

$$
B=\left[\begin{array}{ll}
U_{0} & U_{1}
\end{array}\right]\left[\begin{array}{l}
Z \\
0
\end{array}\right]
$$

with $U=\left[\begin{array}{ll}U_{0} & U_{1}\end{array}\right]$ orthogonal and $Z$ nonsingular. Then $F$ is given by

$$
F=Z^{-1} U_{0}^{\top}\left(X \Lambda X^{-1}-A\right)
$$

Corollary 2.1: ([1], Corollary 1) The eigenvector $x_{i}$ of $A+$ $B F$ corresponding to the assigned eigenvalue $\lambda_{i} \in \mathscr{L}$ must belong to the space

$$
\mathscr{S}_{i} \stackrel{\text { def }}{=} \operatorname{ker}\left[U_{1}^{\top}\left(A-\lambda_{i} I_{n}\right)\right]
$$

the null-space of $U_{1}^{\top}\left(A-\lambda_{i} I_{n}\right)$.

We note that (6) uses a QR factorisation for $B$; Byers and Nash [3] pointed out that $F$ may also be obtained from the singular value decomposition for $B$. Given $B=U S G^{\top}$, we let $U=\left[\begin{array}{ll}U_{0} & U_{1}\end{array}\right]$ and $S G^{\top}=\left[\begin{array}{l}Z \\ 0\end{array}\right]$. They used Corollary 2.1 to obtain a parametric form for the matrix of eigenvectors $X$ satisfying (2) as follows:

Theorem 2.2: ([3]) Assume the eigenvalues in $\mathscr{L}$ are such that $\Lambda$ in (2) is a diagonal matrix. Let $\Sigma_{i}$ be a $n \times m$ basis matrix for $\mathscr{S}_{i}$. Let $\zeta_{(m-1) i+1}, \ldots, \zeta_{m i}$ be the coordinates of the eigenvector $x_{i}$ with respect to $\Sigma_{i}$. The eigenvector $x_{i}$ may be written as

$$
x_{i}=\Sigma_{i} \Xi_{i}, \quad \Xi_{i}=\left[\zeta_{(m-1) i+1}, \ldots, \zeta_{m i}\right]
$$

and the eigenvector matrix $X$ is expressible as

$$
X=X\left(\zeta_{1}, \ldots, \zeta_{n m}\right)=\Sigma \Xi=\left[\Sigma_{1} \ldots \Sigma_{n}\right] \operatorname{diag}\left\{\Xi_{1}, \ldots, \Xi_{n}\right\},
$$

where $\operatorname{diag}\left\{\Xi_{1}, \ldots, \Xi_{n}\right\}$ is an $n m \times n$ block diagonal matrix with $m \times 1$ blocks, so $\zeta$ gives a parameterisation of $X$ and also of $F$.

Theorem 2.2 assumes real eigenvalues; see Section 2.2 of [3] for a comment on how to accommodate complex eigenvalues. Our aim in this paper is to generalise the parametric form for $X$ and $F$ given in Theorem 2.2 to accommodate any admissible Jordan structure $(\mathscr{L}, \mathscr{M}, \mathscr{P})$ for $(A, B)$. Our treatment will explicitly accommodate complex eigenvalues. We begin by noting that for each $i \in\{1, \ldots, v\}$, each $\mathscr{S}_{i}$ has $n$ rows and $n+m$ columns, and as the pair $(A, B)$ is reachable, the dimension of $\mathscr{S}_{i}$ is equal to $m$. For each $i \in\{1,2, \ldots, v\}$, we compute maximal rank matrices $N_{i}$ and $M_{i}$ satisfying

$$
U_{1}^{\top}\left(A-\lambda_{i} I_{n}\right) N_{i}=0, \quad U_{1}^{\top}\left(A-\lambda_{i} I_{n}\right) M_{i}=I_{n-m} .
$$


Then $N_{i}$ is a basis matrix for $\mathscr{S}_{i}$. It follows that, for each odd $i \leq 2 \sigma$, we have $N_{i+1}=\bar{N}_{i}$ because if $\lambda_{i+1}=\bar{\lambda}_{i}$.

For any $\sigma$-conformably ordered admissible Jordan structure $(\mathscr{L}, \mathscr{M}, \mathscr{P})$, we say that an $m \times n$ parameter matrix $K \stackrel{\text { def }}{=}$ $\operatorname{diag}\left\{K_{1}, \ldots, K_{V}\right\}$ is compatible with $(\mathscr{L}, \mathscr{M}, \mathscr{P})$ if: (i) for each $1 \leq i \leq v, K_{i}$ is a matrix of dimension $m \times m_{i}$; (ii) for all $1 \leq i \leq 2 \sigma, K_{i}$ is a complex matrix such that $K_{i}=\bar{K}_{i+1}$, for all odd $i \leq 2 \sigma$, and $K_{i}$ is a real matrix for each $i \geq 2 \sigma$; and (iii) each $K_{i}$ matrix can be partitioned as

$$
K_{i}=\left[\begin{array}{l|l|l|l}
K_{i, 1} & K_{i, 2} & \ldots & K_{i, g_{i}}
\end{array}\right]
$$

where, for $1 \leq k \leq g_{i}$, each $K_{i, k}$ has dimension $m \times p_{i, k}$. In this section we develop our parametric form for the eigenvector matrix $X$ and pole-placing gain matrix $F$ that solve (2) for any admissible eigenstructure $(\mathscr{L}, \mathscr{M}, \mathscr{P})$. Our first task is to obtain a suitable eigenvector matrix. Given a compatible $m \times n$ parameter matrix $K$ for $(\mathscr{L}, \mathscr{M}, \mathscr{P})$, we build eigenvector chains as follows. For each pair $i \in$ $\{1, \ldots, v\}$ and $k \in\left\{1, \ldots, g_{i}\right\}$, build vector chains of length $p_{i, k}$ as follows:

$$
\begin{aligned}
x_{i, k}(1) & =N_{i} K_{i, k}(1), \\
x_{i, k}(2) & =M_{i} U_{1}^{\top} x_{i, k}(1)+N_{i} K_{i, k}(2), \\
& \vdots \\
x_{i, k}\left(p_{i, k}\right) & =M_{i} U_{1}^{\top} x_{i, k}\left(p_{i, k}-1\right)+N_{i} K_{i, k}\left(p_{i, k}\right) .
\end{aligned}
$$

From these column vectors we construct the matrices

$$
\begin{aligned}
X_{i, k} & \stackrel{\text { def }}{=}\left[x_{i, k}(1)\left|x_{i, k}(2)\right| \ldots \mid x_{i, k}\left(p_{i, k}\right)\right] \\
X_{i} & \stackrel{\text { def }}{=}\left[X_{i, 1}\left|X_{i, 2}\right| \ldots \mid X_{i, g_{i}}\right] \\
X_{K} & \stackrel{\text { def }}{=}\left[X_{1}\left|X_{2}\right| \ldots \mid X_{V}\right]
\end{aligned}
$$

of dimensions $n \times p_{i, k}, n \times m_{i}$ and $n \times n$, respectively. Finally we obtain the feedback gain matrix

$$
F_{K} \stackrel{\text { def }}{=} Z^{-1} U_{0}^{\top}\left(X_{K} \Lambda X_{K}^{-1}-A\right)
$$

Given the origins of this method in the classic paper [1], we shall refer to the parametric formulae (18)-(19) as the extended Kautsky-Nichols-van Dooren parametric form for $X$ and $F$. We are now ready to present the main result of this paper.

Theorem 2.3: Let $(\mathscr{L}, \mathscr{M}, \mathscr{P})$ be an admissible Jordan structure for $(A, B)$ and let $K$ be a compatible parameter matrix. Then for almost all choices of $K$, the matrix $X_{K}$ in (18) is invertible, i.e., $X_{K}$ is invertible for every choice of $K$ except those lying in a set of measure zero. The set of all real feedback matrices $F_{K}$ such that the closed-loop matrix $A+B F_{K}$ has Jordan structure given by $(\mathscr{L}, \mathscr{M}, \mathscr{P})$ is parameterised in $K$ by (19), where $X_{K}$ is obtained with a parameter matrix $K$ such that $X_{K}$ is invertible.

Proof: The proof will be carried out in three steps. First, we show that if $X_{K}$ and $F_{K}$ are given by (18) and (19) respectively, then (2) is satisfied, provided $X_{K}$ is invertible. Second, we show that the parametrisation given in (19) is exhaustive, i.e., for every feedback matrix $F_{K}$ and nonsingular eigenvector matrix $X_{K}$ satisfying (2), there exists a compatible parameter matrix $K$ such that $X_{K}$ and $F_{K}$ can be recovered from (18) and (19), respectively. Finally, we prove that for almost every compatible parameter $K$, the matrix $X_{K}$ in (18) is non-singular.

We start proving the first point. Let $K$ be a compatible input parameter matrix as in (12), and for each $i \in\{1, \ldots, v\}$ and $k \in\left\{1, \ldots, g_{i}\right\}$, let $X_{i, k}, X_{i}$ and $X_{K}$ be constructed as in (16)(18) respectively. Then the column vectors of $X_{i, k}$ satisfy (13)-(15) by construction. Thus

$$
\begin{aligned}
U_{1}^{\top}\left(A-\lambda_{i} I_{n}\right) x_{i, k}(1)= & U_{1}^{\top}\left(A-\lambda_{i} I_{n}\right) N_{i} K_{i, k}(1)=0, \text { (20) } \\
U_{1}^{\top}\left(A-\lambda_{i} I_{n}\right) x_{i, k}(2)= & U_{1}^{\top}\left(A-\lambda_{i} I_{n}\right) M_{i} U_{1}^{\top} x_{i, k}(1) \\
& +U_{1}^{\top}\left(A-\lambda_{i} I_{n}\right) N_{i} K_{i, k}(2) \\
= & U_{1}^{\top} x_{i, k}(1) \\
\vdots & \\
U_{1}^{\top}\left(A-\lambda_{i} I_{n}\right) x_{i, k}\left(p_{i, k}\right)= & U_{1}^{\top}\left(A-\lambda_{i} I_{n}\right) M_{i} U_{1}^{\top} x_{i, k}\left(p_{i, k}-1\right) \\
& +U_{1}^{\top}\left(A-\lambda_{i} I_{n}\right) N_{i} K_{i, k}\left(p_{i, k}\right) \\
= & U_{1}^{\top} x_{i, k}\left(p_{i, k}-1\right) .
\end{aligned}
$$

Hence the vectors $x_{i, k}(1), \ldots, x_{i, k}\left(p_{i, k}\right)$ form a chain of generalised eigenvectors for the matrix $U_{1}^{\top}\left(A-\lambda_{i} I_{n}\right)$, and so

$$
U_{1}^{\top}\left(A X_{i, k}-X_{i, k} J_{k}\left(\lambda_{i}\right)\right)=0 \text {. }
$$

Thus,

$$
U_{1}^{\top}\left(A X_{i}-X_{i} J\left(\lambda_{i}\right)\right)=0
$$

and finally we have

$$
U_{1}^{\top}\left(A X_{K}-X_{K} \Lambda\right)=0 .
$$

Assume $X_{K}$ is non-singular and obtain $F_{K}$ from (19). We note that $F_{K}$ is a real matrix because for each odd $i \in\{1, \ldots, 2 \sigma\}$, we have $\lambda_{i+1}=\bar{\lambda}_{i}$ and $X_{i+1}=\bar{X}_{i}$. Multiplying through by $B=U_{0} Z$ we obtain

$$
B F_{K}=X_{K} \Lambda X_{K}^{-1}-A
$$

and hence $X_{K}$ and $F_{K}$ satisfy (2).

Next we show that the above parametrisation is exhaustive. We let $X$ and $F$ be any pair of matrices satisfying (2) such that the eigenstructure of $A+B F$ is described by $(\mathscr{L}, \mathscr{M}, \mathscr{P})$. Then we can decompose $X$ into block matrices

$$
X=\left[X_{1}\left|X_{2}\right| \ldots \mid X_{V}\right]
$$

where for $i \in\{1, \ldots, v\}$,

$$
X_{i}=\left[X_{i, 1}\left|X_{i, 2}\right| \ldots \mid X_{i, k}\right]
$$

and for $k \in\left\{1, \ldots, g_{i}\right\}$

$$
X_{i, k}=\left[x_{i, k}(1)\left|x_{i, k}(2)\right| \ldots \mid x_{i, k}\left(p_{i, k}\right)\right]
$$

and the vectors $x_{i, k}(1), x_{i, k}(2), \ldots, x_{i, k}\left(p_{i, k}-1\right)$ form a chain of generalised eigenvectors for $A+B F$ with respect to $\lambda_{i}$. Hence, we have

$$
\begin{aligned}
\left(A+B F-\lambda_{i} I_{n}\right) x_{i, k}(1) & =0 \\
\left(A+B F-\lambda_{i} I_{n}\right) x_{i, k}(2) & =x_{i, k}(1) \\
& \vdots \\
\left(A+B F-\lambda_{i} I_{n}\right) x_{i, k}\left(p_{i, k}\right) & =x_{i, k}\left(p_{i, k}-1\right)
\end{aligned}
$$


Thus from (30) we have

$$
\begin{aligned}
\left(A-\lambda_{i} I_{n}\right) x_{i, k}(1) & =-B F x_{i, k}(1) \\
\Rightarrow U_{1}^{\top}\left(A-\lambda_{i} I_{n}\right) x_{i, k}(1) & =-U_{1}^{\top} B F x_{i, k}(1)=0
\end{aligned}
$$

as $U_{1}^{\top} B=0$. Hence there exists a compatible parameter matrix $K_{i, k}(1)$ of dimension $m \times 1$ such that (13) holds. Also from (31) we have

$$
\begin{aligned}
\left(A-\lambda_{i} I_{n}\right) x_{i, k}(2) & =-B F x_{i, k}(2)+x_{i, k}(1) \\
\Rightarrow U_{1}^{\top}\left(A-\lambda_{i} I_{n}\right) x_{i, k}(2) & =-U_{1}^{\top} B F x_{i, k}(2)+U_{1}^{\top} x_{i, k}(1) \\
& =U_{1}^{\top} x_{i, k}(1)
\end{aligned}
$$

and hence (14) holds for some parameter matrix $K_{i, k}(2)$. Similarly we can use (32) to obtain the parameter $K_{i, k}\left(p_{i, k}\right)$ such that (15) holds. Combining these parameters we obtain an $m \times p_{i, k}$ parameter matrix $K_{i, k}$; combining these for all $k \in\left\{1, \ldots, g_{i}\right\}$ we obtain a parameter matrix $K_{i}$ of dimension $m \times m_{i}$, and finally combining these for all $i \in\{1, \ldots, v\}$ we obtain a parameter matrix $K$ of dimension $m \times n$. Further it is clear that if $\lambda_{i}$ and $\lambda_{i+1}$ are such that $\bar{\lambda}_{i+1}=\lambda_{i}$ then $\bar{K}_{i+1}=K_{i}$. Hence applying the procedure in (13)-(15) with this parameter matrix $K$ will yield $X_{K}=X$, and applying (19) with this $X_{K}$ yields $F_{K}=F$.

Finally let us prove the third point. We let $N_{i} \stackrel{\text { def }}{=}$ $\left[n_{i, 1}|\ldots| n_{i, m}\right]$ be an orthonormal basis matrix for $\mathscr{S}_{i}$, and for each $i \in\{1, \ldots, v\}$ and $k \in\left\{1, \ldots, g_{i}\right\}$, we introduce vectors

$$
\begin{aligned}
v_{i, k}(1) & =n_{i, k} \\
v_{i, k}(2) & =M_{i} U_{1}^{\top} v_{i, k}(1) \\
& \vdots \\
v_{i, k}\left(p_{i, k}\right) & =M_{i} U_{1}^{\top} v_{i, k}\left(p_{i, k}-1\right)
\end{aligned}
$$

and using these we obtain matrices

$$
\begin{aligned}
V_{i, k} & \stackrel{\text { def }}{=}\left[v_{i, k}(1)\left|v_{i, k}(2)\right| \ldots \mid v_{i, k}\left(p_{i, k}\right)\right], \\
V_{i} & \stackrel{\text { def }}{=}\left[V_{i, 1}\left|V_{i, 2}\right| \ldots \mid V_{i, g_{i}}\right], \\
V & \stackrel{\text { def }}{=}\left[V_{1}\left|V_{2}\right| \ldots \mid V_{v}\right]
\end{aligned}
$$

of dimensions $n \times p_{i, k}, n \times m_{i}$, and $n \times n$, respectively. Then we have $\operatorname{rank}(V)=n$, because if the rank is strictly smaller than $n$, then no parameter matrix $K$ exists to construct $F_{K}$ in (19) that will deliver the desired closed-loop eigenstructure. On the other hand, we showed that the parameterisation given by (19) is exhaustive and $(\mathscr{L}, \mathscr{M}, \mathscr{P})$ are an admissible Jordan structure. Hence, this means in particular that no feedback matrix can deliver the required closed-loop eigenstructure. This means that the pair $(A, B)$ is not reachable, which leads to a contradiction.

Next let $K$ be any compatible parameter matrix for $(\mathscr{L}, \mathscr{M}, \mathscr{P})$, let $X=V K$ and assume $X$ is singular, i.e. $\operatorname{rank}(X) \leq n-1$. This means that one column of the matrix

$$
\begin{aligned}
& {\left[v_{1,1}(1) K_{1,1}(1) \ldots v_{1,1}\left(p_{1,1}\right) K_{1,1}\left(p_{1,1}\right) \ldots\right.} \\
& \left.\quad \ldots v_{v, g_{v}}(1) K_{v, g_{v}}(1) \ldots v_{v, g_{v}}\left(p_{v, g_{v}}\right) K_{v, g_{v}}\left(p_{v, g_{v}}\right)\right]
\end{aligned}
$$

is linearly dependent upon the remaining ones. For the sake of argument, assume this is the last column. This means that there exist coefficients $\left\{\alpha_{i, k, l}: 1 \leq i \leq v, 1 \leq k \leq g_{i}, 1 \leq l \leq\right.$ $\left.p_{i, k}\right\}$ (not all equal to zero) for which

$$
\begin{aligned}
v_{v, g_{v}}\left(p_{v, g_{v}}\right) K_{v, g_{v}}\left(p_{v, g_{v}}\right)= & \sum_{i=1}^{v-1} \sum_{k=1}^{g_{i}} \sum_{l=1}^{p_{i, k}} \alpha_{i, k, l} v_{i, g_{i}}(l) \\
& +\sum_{k=1}^{g_{v}-1} \sum_{l=1}^{p_{v, k}} \alpha_{v, k, l} v_{v, k}(l) \\
& +\sum_{l=1}^{p_{v, g_{v}}-1} \alpha_{v, g_{v}, l} v_{v, g_{v}}(l) .
\end{aligned}
$$

This implies that $\operatorname{rank}(V K)=n$ may fail only when $K_{V, g_{v}}\left(p_{v, g_{v}}\right)$ lies on an $(m-1)$-dimensional hyperplane in the $m$-dimensional parameter space. Thus the set of compatible parameter matrices $K$ that can lead to a loss of rank in $X_{K}$ is given by the union of a finite number of hyperplanes of dimension at most $m-1$ within the parameter space. Since hyperplanes have measure zero with respect to Lebesgue measure on the $m$-dimensional parameter space, we conclude the set of parameter matrices $K$ leading to singular $X_{K}$ has zero Lebesgue measure.

\section{Minimum Gain Pole Placement}

We utilise the parametric form introduced in the previous section to consider the problem of minimising the norm of the gain matrix $F$. More precisely, we consider the unconstrained optimisation problem

$$
(\mathscr{P}): \min _{K}\left\|F_{K}\right\|_{\mathrm{FRO}}^{2}
$$

where $F_{K}$ in (19) arises from any compatible parameter matrix $K$. Problem $\mathscr{P}$ may be addressed via a gradient search employing the first and second order derivatives of $\left\|F_{K}\right\|_{\mathrm{FRO}}^{2}$. From these the gradient and Hessian matrices are easily obtained, and unconstrained nonlinear optimisation methods can then be used to seek local minima. Such an optimisation approach was considered in [16], but only for $\mathscr{L}$ with distinct eigenvalues. The method presented in this paper can accommodate any desired admissible eigenstructure.

\section{ILLUSTRATIVE EXAMPLES}

In this section, we compare the algorithm presented in this paper with the methods given in [11], [14], [15].

Example 4.1: We consider Example 1 in [15], and seek to design a deadbeat controller, which can be achieved with one Jordan mini-block of dimension two, and two blocks of dimension 1. The method of [15] aims to minimise the Frobenius norm of the gain matrix and delivers the feedback matrix

$$
F=-\left[\begin{array}{cccc}
0.5 & 0.5 & -0.0889 & 0.4556 \\
0.5 & -0.5 & -0.1111 & -0.0556 \\
0 & 0 & 0.2444 & 0.6222
\end{array}\right]
$$

yielding a normalised eigenvector matrix $X$ with $\kappa_{\mathrm{FRO}}(X)=$ 431.36 and gain matrix $\|F\|_{\mathrm{FRO}}=1.2953$. Applying our method, we also obtain the matrix $F$. 
Example 4.2: We consider the example 3.1 in [11] with $n=$ 4 and $m=2$. The method presented in this paper produces the feedback matrix

$$
F=\left[\begin{array}{cccc}
2.0000 & 0.0000 & -0.0000 & -2.0000 \\
0.0000 & -2.0000 & 2.0000 & -0.0000
\end{array}\right],
$$

whose Frobenius norm is equal to 4 . This result ties up with the method in [11].

Example 4.3: Now we study the example in [14] with $n=9$ and $m=4$ in which a gain matrix is sought to place all the closed-loop poles at $\lambda=-0.55$. In this example, the controllability indexes are $\{3,2,2,2\}$. Hence, a gain matrix $F$ can be obtained such that $(A+B F-\lambda I)^{l}=0$ for any $l$ between 3 and 9 .

In this example, the maximum iteration is chosen as 5000 and the initial condition is given as

$$
\begin{gathered}
K(0)=\operatorname{diag}\left\{\left[\begin{array}{c}
-1 \\
-2 \\
0 \\
2
\end{array}\right],\left[\begin{array}{c}
1 \\
-1 \\
0 \\
1
\end{array}\right],\left[\begin{array}{c}
1 \\
0 \\
-1 \\
-1
\end{array}\right],\left[\begin{array}{c}
1 \\
-1 \\
1 \\
0
\end{array}\right],\right. \\
\left.\left[\begin{array}{c}
-1 \\
3 \\
2 \\
0
\end{array}\right],\left[\begin{array}{c}
3 \\
0 \\
-2 \\
2
\end{array}\right],\left[\begin{array}{c}
-4 \\
1 \\
0 \\
2
\end{array}\right],\left[\begin{array}{c}
1 \\
0 \\
3 \\
-1
\end{array}\right],\left[\begin{array}{c}
-2 \\
1 \\
1 \\
2
\end{array}\right]\right\} .
\end{gathered}
$$

For the case $(A-\lambda I+B F)^{3}=0$, the method in [14] produces a feedback matrix $F_{1}$ with $\left\|F_{1}\right\|_{\text {FRO }}=1.5 \times 10^{7}$. Our method based on the Klein-Moore parametric form in [11] produces a gain matrix $F_{2}$ with $\left\|F_{2}\right\|_{\mathrm{FRO}}=4.4 \times 10^{5}$.

The method given in this paper via the extended KautskyNichols-van Dooren parametric form gives a gain matrix $F_{3}$ with $\left\|F_{3}\right\|_{\text {FRO }}=1.3 \times 10^{4}$ where

$$
\begin{gathered}
F_{3}=10^{4} *\left[\begin{array}{ccccc}
0.1105 & -0.0004 & 0.0007 & 0.1075 \\
-1.2226 & -0.0008 & 0.0001 & 0.0096 \\
0.0027 & 0.0680 & 0.0500 & -0.0032 \\
0.0869 & -0.0004 & 0.0008 & 0.1322 \\
& & & & \\
& & & & \\
-0.0000 & 0.0004 & 0.0006 & -0.0001 & -0.0018 \\
-0.0000 & 0.0004 & -0.0042 & -0.0030 & -0.0005 \\
-0.0101 & 0.1377 & 0.2159 & 0.0149 & -0.0065 \\
-0.0000 & 0.0006 & 0.0008 & -0.0002 & -0.0021
\end{array}\right]
\end{gathered}
$$

For the case $(A-\lambda I+B F)^{5}=0$, the solution $F_{4}$ in [14] is such that $\left\|F_{4}\right\|_{\mathrm{FRO}}=9.2 \times 10^{2}$. The procedure in [11] results in a gain matrix $F_{5}$ with $\left\|F_{5}\right\|_{\mathrm{FRO}}=6.8 \times 10^{2}$. Using our scheme, we obtain a gain matrix $F_{6}$ with $\left\|F_{6}\right\|_{\text {FRO }}=7.3 \times 10^{2}$ where

$$
\begin{aligned}
& F_{6}=\left[\begin{array}{cccc}
254.3428 & -2.3338 & 2.1648 & -247.0867 \\
-17.2078 & -76.7139 & 65.2416 & -3.3914 \\
-3.1697 & -124.2937 & 364.2689 & -0.1693 \\
194.1004 & -3.8408 & 3.1736 & 152.1953
\end{array}\right. \\
& \left.\begin{array}{ccccc}
-0.0071 & 0.0551 & -1.7144 & -2.2267 & 5.5261 \\
-6.8858 & 7.4137 & -43.4673 & -76.1844 & -38.8967 \\
-131.8946 & 116.9070 & 339.8278 & -184.8227 & -13.1262 \\
-0.2134 & 0.2976 & -1.4964 & -3.8142 & -1.3832
\end{array}\right] .
\end{aligned}
$$

\section{CONCLUSiON}

We have extended the classic pole placement method of Kautsky, Nichols and van Dooren to obtain a parametric form for the problem of exact pole placement that can accommodate any desired eigenstructure with arbitrary multiplicities. The method places no restrictions on the set of poles that can be assigned, nor their multiplicities, other than those implied by the constraints of the Rosenbrock theorem. The method provides an interesting parallel to the parametric formula given in [11] that also achieved arbitrary pole placement, but was derived from the Klein-Moore parametric form.

Examples were given to show that this method delivers pole placement with considerably less matrix gain than the alternative in [14]. The comparisons against the method of [11] showed similar performance in minimising the matrix gain. Future work will consider whether either of these two optimal pole placement methods enjoys any significant performance advantages over the other, with respect to minimising the matrix gain.

\section{APPENDIX}

Here we consider the first and second derivatives of $f$ in (38). We define

$$
\chi_{i} \stackrel{\text { def }}{=} \begin{cases}\mathfrak{R e}\left\{K_{i}\right\} & i \in\{1, \ldots, 2 \sigma\} \text { odd, } \\ \mathfrak{I m}\left\{K_{i-1}\right\} & i \in\{1, \ldots, 2 \sigma\} \text { even, } \\ K_{i} & i \in\{2 \sigma+1, \ldots, v\} .\end{cases}
$$

Let

$$
H_{K}=\mathfrak{R e}\left\{X_{K}\right\} .
$$

Define $\chi_{i, k}(l, r)$ as the $r$-th entry of $\chi_{i, k}(l)$. We compute the derivative of $Y_{p, q}$ with respect to $\chi_{i, k}$. We have

$$
\frac{\partial H_{p, q}}{\partial \chi_{i, k}(l, r)}=0
$$

for $p \in\{1, \ldots, 2 \sigma\}$ with $p \neq i, p \neq i+\sigma, p+\sigma \neq i$ and $p \in$ $\{2 \sigma+1, \ldots, v\}$ with $p \neq i$. Define

$$
P(i, l) \stackrel{\text { def }}{=} \begin{cases}\left\{M_{i} U_{1}^{\top}\right\}^{l} N_{i} & \text { if } l \geq 0, \\ 0 & \text { otherwise. }\end{cases}
$$

For each $i \in\{1, \ldots, \sigma\}, k \in\left\{1, \ldots, g_{i}\right\}, h, l \in\left\{1, \ldots, p_{i, k}\right\}$ and $r \in\{1, \ldots, m\}$ we find

$$
\begin{aligned}
\frac{\partial H_{i, k}(h)}{\partial \chi_{i, k}(l, r)} & =\mathfrak{R e}\{P(i, h-l)\}(r), \\
\frac{\partial H_{i+\sigma, k}(h)}{\partial \chi_{i, k}(l, r)} & =\mathfrak{I m}\{P(i, h-l)\}(r), \\
\frac{\partial H_{i, k}(h)}{\partial \chi_{i+\sigma, k}(l, r)} & =-\mathfrak{I m}\{P(i, h-l)\}(r), \\
\frac{\partial H_{i+\sigma, k}(h)}{\partial \chi_{i+\sigma, k}(l, r)} & =\mathfrak{R e}\{P(i, h-l)\}(r) .
\end{aligned}
$$

For each $i \in\{2 \sigma+1, \ldots, v\}, k \in\left\{1, \ldots, g_{i}\right\}, h, l \in$ $\left\{1, \ldots, p_{i, k}\right\}$ and $r \in\{1, \ldots, m\}$ we have

$$
\frac{\partial H_{i, k}(h)}{\partial \chi_{i, k}(l, r)}=P(i, h-l)(r) \text {. }
$$


Let $Y_{K}=H_{K}^{-1}$. Using the well-known formula $\frac{\partial Y_{K}}{\partial \chi_{i, k}(l, r)}=$ $-Y_{K} \frac{\partial H_{K}}{\partial \chi_{i, k}(l, r)} Y_{K}$, we compute the first and second derivatives of $\left\|F_{K}\right\|_{\text {FRO }}^{2}$ as

$$
\frac{\partial\left\|F_{K}\right\|_{\mathrm{FRO}}^{2}}{\partial \chi_{i, k}(l, r)}=2 \operatorname{trace}\left(F_{K}^{\top} Q_{K}(i, k, l, r)\right)
$$

and

$$
\begin{gathered}
\frac{\partial^{2}\left\|F_{K}\right\|_{\mathrm{FRO}}^{2}}{\partial \chi_{i_{1}, k_{1}}\left(l_{1}, r_{1}\right) \partial \chi_{i_{2}, k_{2}}\left(l_{2}, r_{2}\right)} \\
=2 \operatorname{trace}\left(Q_{K}\left(i_{2}, k_{2}, l_{2}, r_{2}\right)^{\top} Q_{K}\left(i_{1}, k_{1}, l_{1}, r_{1}\right)\right. \\
-F_{K}^{\top} Q_{K}\left(i_{2}, k_{2}, l_{2}, r_{2}\right) \frac{\partial H_{K}}{\partial \chi_{i_{1}, k_{1}}\left(l_{1}, r_{1}\right)} Y_{K} \\
\left.-F_{K}^{\top} Q_{K}\left(i_{1}, k_{1}, l_{1}, r_{1}\right) \frac{\partial H_{K}}{\partial \chi_{i_{2}, k_{2}}\left(l_{2}, r_{2}\right)} Y_{K}\right)
\end{gathered}
$$

where

$$
\begin{aligned}
& Q_{K}(i, k, l, r)= \\
& Z^{-1} U_{0}^{\top}\left(\frac{\partial H_{K}}{\partial K_{i, k}(l, r)} \Lambda Y_{K}-H_{K} \Lambda Y_{K} \frac{\partial H_{K}}{\partial K_{i, k}(l, r)} Y_{K}\right) . \\
& \text { REFERENCES }
\end{aligned}
$$

\section{REFERENCES}

[1] J. Kautsky, J. N.K. Nichols and P. Van Dooren, Robust Pole Assignment in Linear State Feedback, International Journal of Control, vol. 41, pp. 1129-1155, 1985.

[2] H. H. Rosenbrock, State-Space and Multioariable Theory. New York: Wiley, 1970.

[3] R. Byers and S. G. Nash, Approaches to robust pole assignment, International Journal of Control, vol. 49, pp. 97-117, 1989.
[4] S.P. Bhattacharyya and E. de Souza, Pole assignment via Sylvester's equation, Systems \& Control Letters, vol. 1(4), pp. 261-263, 1982.

[5] M.M. Fahmy and J. O'Reilly, Eigenstructure Assignment in Linear Multivariable Systems-A Parametric Solution, IEEE Transactions on Automatic Control, vol. 28, pp. 990-994, 1983.

[6] E. Chu, Pole assignment via the Schur form, Systems \& Control Letters vol. 56, pp, 303-314, 2007.

[7] M. Ait Rami, S.E. Faiz, and A. Benzaouia, Robust Exact Pole Placement via an LMI-Based Algorithm, IEEE Transactions on Automatic Control, Vol. 54(2) pp. 394-398, 2009.

[8] R. Schmid, T. Nguyen and A. Pandey, Optimal Pole placement with Moore's algorithm, in Proceedings 1st IEEE Australian Control Conference, Melbourne, Australia, 2011.

[9] R. Schmid, A. Pandey and T. Nguyen, Robust Pole placement with Moore's algorithm, IEEE Transactions on Automatic Control vol. 59, pp. 500-505, 2014.

[10] B.C. Moore, On the Flexibility Offered by State Feedback in Multivariable systems Beyond Closed Loop Eigenvalue Assignment, IEEE Transactions on Automatic Control, vol. 21(5), pp. 689-692, 1976.

[11] R. Schmid, L. Ntogramatzidis, T. Nguyen and A. Pandey, Arbitrary pole placement with minimum gain, Proceedings 21st Mediterranean Conference on Control and Automation, Crete, 2013.

[12] R. Schmid, L. Ntogramatzidis, T. Nguyen and A. Pandey, Robust repeated pole placement, to appear Proceedings 3rd IEEE Australian Control Conference, Perth, 2013.

[13] G. Klein and B.C. Moore, Eigenvalue-Generalized Eigenvector Assignment with State Feedback, IEEE Transactions on Automatic Control, vol. 22(1), pp. 141-142, 1977.

[14] A. Linnemann, An algorithm to compute state feedback matrices for multi-input deadbeat control, Systems \& Control Letters vol. 25, pp, 99-102, 1995.

[15] M. Ataei and A. Enshaee, Eigenvalue assignment by minimal statefeedback gain in LTI multivariable systems, International Journal of Control, vol. 84, pp. 1956-1964, 2011.

[16] H.K. Tam and J. Lam, Newton's approach to gain-controlled robust pole placement, IEE Proc.-Control Theory Applications. 144(5), pp. 439-446, 1997. 